\title{
Chronic-pharma: New Platform for Chronic Patients Pharmacotherapy Optimization
}

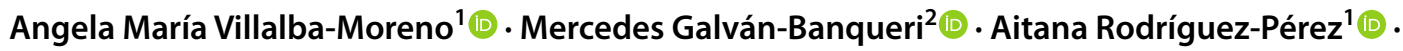 \\ María Dolores Toscano-Guzmán ${ }^{1}$ (i) . Clara López-Hermoso ${ }^{1}$ (D) Susana Sánchez-Fidalgo ${ }^{3}$ (i) . \\ Bernardo Santos-Ramos ${ }^{1}\left[\right.$ ] Eva Rocío Alfaro-Lara ${ }^{1}[$
}

Received: 12 May 2021 / Accepted: 12 February 2022 / Published online: 28 February 2022

(c) The Author(s) 2022

\begin{abstract}
We describe the technological development of a web platform named CHRONIC-PHARMA that integrates three prescription support tools for patients with chronic diseases: Anticholinergic Burden Calculator (ABC), LESS-CHRON criteria and TRIGGER-CHRON. They focus on the optimization and evaluation of pharmacotherapy in patients with chronic diseases, resulting in a useful, single platform that can facilitate the review of pharmacotherapy and improve the safety of chronically ill patients. This is achieved by estimating and reducing the anticholinergic risk (ABC), detecting opportunities for deprescribing drugs and monitoring its success (LESS-CHRON criteria), as well as calculating the risk of adverse drug events (TRIGGER-CHRON). The platform is freely accessible online (https://chronic-pharma.com/) as well as through a mobile application, and therefore easily accessible among the healthcare community.
\end{abstract}

Keywords Clinical decision support system · Technological development · Chronic patient · Deprescribing · Trigger tool · Anticholinergic burden

\section{Introduction}

The high prevalence of chronic diseases is changing the profile of patients who require healthcare. Patients with chronic diseases are a group of special interest since their care is predominated by complex polypharmacy, which is a prime risk factor for inappropriate prescribing and adverse drug reactions and events. Implementing an appropriate treatment plan for these patients is a difficult and complex process. They require a comprehensive quality approach, with appropriate pharmacotherapeutic optimization mediated by prescription support tools which standardize the

This article is part of the Topical Collection on Implementation Science \& Operations Management

Susana Sánchez-Fidalgo

fidalgo@us.es

1 Pharmacy Service, University Hospital Virgen del Rocío, Seville, Spain

2 Pharmacy Service, University Hospital Virgen de Valme, Seville, Spain

3 Department of Preventive Medicine and Public Health, University of Seville, Seville, Spain recommendations of pharmacotherapy, considering the specific situation of the patient [1]. Creating such prescription support tools is an additional challenge; they need to be designed, built, validated, and tested robustly so that they can help to perform complex medication reviews.

There are widely accepted tools that have been consolidated as guidelines to help in the global review of treatment in elderly patients: STOPP-START criteria or the Beers criteria $[2,3]$. They are fundamental tools to be considered in any digital platform for the global review of treatment in elderly patients. Indeed, a computer-generated STOPP/ START is currently being investigated to examine the impact of criteria on incident ADRs (SENATOR) and drug-related hospitalizations (OPERAM) [4].

In recent years, other tools have been published that have also added value to the pharmacotherapeutic review process from more specific perspectives in complex chronic patients. This is the case of anticholinergic scales or deprescribing strategies, between others. In this case, the development of its concepts has often gone hand in hand with its technological implementation, since the advantage of electronic prescription support systems in the medication review process has been demonstrated $[5,6]$. 
The risk of developing adverse anticholinergic effects is a significant issue in polypharmacy. Tools to identify this risk are named anticholinergic scales [7, 8]. Our research group (http://chronicpharma.es/) has developed a tool which calculates the anticholinergic burden based on ten anticholinergic scales. The scales have been firstly identified in a systematic review carried out in chronic patients with multimorbidity, with the aim to be used in clinical settings [9]. The tool was named the Anticholinergic Burden Calculator (ABC), and accessible online via a web portal software program (www.anticholinergicscales.es/) [10].

In recent years, the concept of deprescribing has been raised worldwide as an essential review process to reduce the therapeutic burden (drug burden) [11, 12]. Some guidelines are aimed at deprescribing specific groups of drugs such as benzodiazepines or proton pump inhibitors (for example, Canadian deprescribing guidelines) [13, 14], or to identify patients whose treatments contain drugs to be deprescribed as suggested by deprescribing criteria focused on different type of patients $[15,16]$. The LESS-CHRON criteria (List of Evidence baSed depreScribing for CHRONic patients) are a comprehensive and standardized methodology to identify clinical situations for deprescribing drugs in chronic patients with multimorbidity, considering life prognosis and providing health variables to value the safety of the process. They were translated into English using a transcultural methodology [17], and validated by a reliability study [18].

The trigger tool methodology was developed by the Institute for Healthcare Improvement as a low-resource option to detect adverse events within hospital settings [19]. Trigger tools involve the application of various screening criteria to guide the medical review process in the identification of adverse events [20]. They seem to be the most efficient and cost-effective singular method for detecting harm associated with health care, which is why numerous studies using the trigger method to measure adverse drug events (ADEs) rates in health care organizations have been published [21]. TRIGGER-CHRON has recently been developed as a tool that uses the trigger method for elderly patients with multimorbidity. The development was carried out in two consecutives phases. Firstly, the most common triggers for that population were selected [22]. Secondly, 32 of that 51 previously identified, were selected based on their positive predictive value, obtaining the TRIGGER-CHRON [23].

Healthcare is facing the emergence of a new range of systems and applications using electronic communication. The use of health applications has recently seen rapid and steady growth. In 2014, compared to the previous year, the time spent on applications in the health category increased by $89 \%$ [24].

Thus, the objective of this work is to describe the technological development of a platform that integrates the three tools developed by our research group: the ABC Calculator, the LESS-CHRON Criteria and the TRIGGER-CHRON criteria.

\section{Methods and study design}

The web platform and mobile app development was conducted from November 2018 to June 2020.

Previously, each of the tools (ABC, LESS-CHRON and TRIGGER-CHRON) were developed within a multidisciplinary team of hospital pharmacists, internal medicine specialists and general practitioners [10, 17, 22]. To integrate the knowledge generated into the web format, a research group was formed with the hospital pharmacists responsible for each tool, a total of six professionals. An external company with experience in transferring research results to the market was contracted. The research group directed and supervised the company in the design of the website and app, the development of the content and the prototype, ensuring that the development of the platform met the objectives established. Finally, the researchers conducted the usability evaluation of the provisional version before completing the process (Fig. 1).

The design and visual appearance of the app was the same as that used for the web platform. It was developed for the Android platform.

In order to validate the usability, each investigator responsible for the tool performed separate performance tests on simulated patients with multiple scenarios, followed by a second investigator. Finally, the research group reviewed the final version for enhancements and bug corrections.

\section{Results}

The platform created is called CHRONIC-PHARMA. It is available via a web page (https://chronic-pharma.com/) and a mobile health application available on the Google Play Store. It has integrated three tools that can be used independently in each registered patient: estimating the anticholinergic risk and assessing possible interventions for its reduction (ABC), detecting drugs to be deprescribed and monitoring its success (LESS-CHRON) and calculating the risk that a patient has of having an ADE (TRIGGER-CHRON). (Fig. 2). All three have been developed using robust systematic review methodologies and structured clinical consensus [10, 17, 22]. ABC includes previously published and validated scales identified in a systematic review [9]. LESS-CHRON and final TRIGGER-CHRON are in the process of validation in patients, which is the most expected study for a tool of this category; however, both has been applied in a theorical way, validating its utility $[18,23]$. In relation to the complexity 


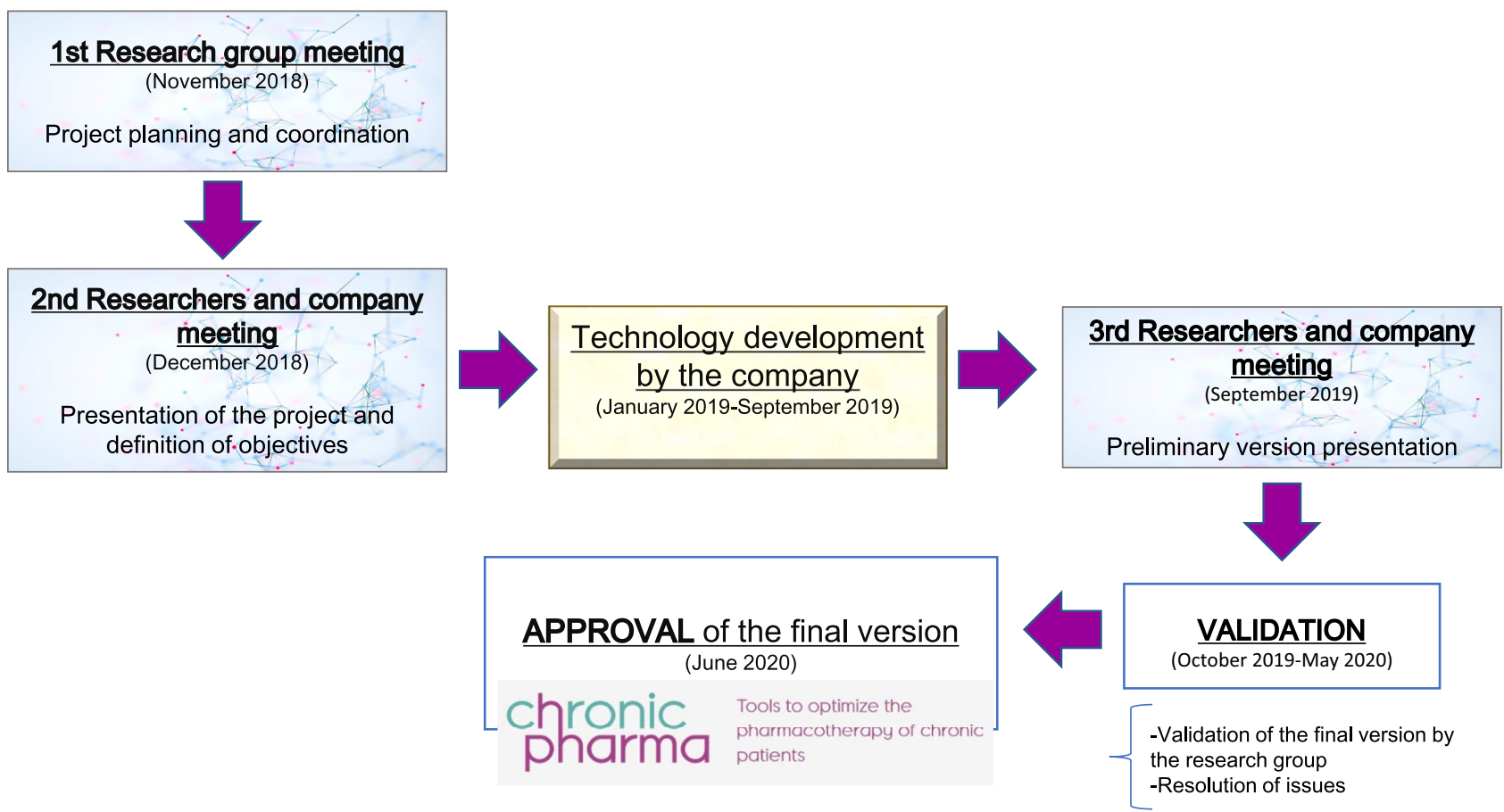

Fig. 1 Diagram of the evolution of the project phases "CHRONIC-PHARMA" web

when adapting the tools to web format, it should be noted that $\mathrm{ABC}$ differs from the LESS-CHRON and TRIGGERCHRON tools in the modus operandi it requires, as well as in the expression of the results. For example, DBI requires a calculation considering daily dosage of drugs [25],
LESS-CHRON and TRIGGER-CHRON serve as a guide for the preparation of a final recommendation report that does not establish weight differences between the prescribed drugs, but it adheres to pre-specified criteria that the user must decide if they are met or not in the target patient.

Fig. 2 Homepage of CHRONIC-PHARMA-web

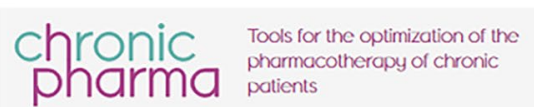

patients
米

Sign Up Login
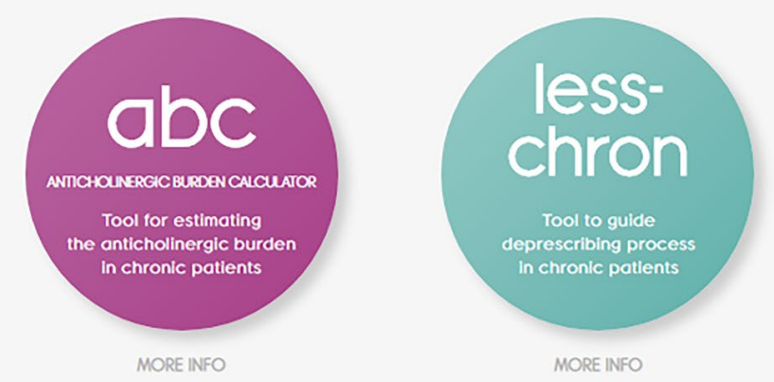

MORE INFO 
The web/app platform is free access and can be used in both English and Spanish. We therefore hope that this platform can be disseminated and used by healthcare professionals at any level of care. The platform structure is simple, with users filling in the data that it asks for depending on the characteristics and pharmacotherapy of each patient, and not having to review each of the criteria or drugs.

Each tool is accompanied with an explanation on how to use it, as well as its interpretation.

1. ABC. The anticholinergic burden is calculated based on the nine anticholinergic scales and Drug Burden Index (DBI), after introducing anticholinergic drugs and the total daily dose required for DBI. The burden is reported quantitatively and the risk for each scale is qualitatively color-coded (null, low, medium, and high). The individual drugs involved are also reported along with their anticholinergic potential given by each scale. Due to the variability among the anticholinergic scales, $\mathrm{ABC}$ calculator is intended to be an aid to the calculation by offering all the possibilities. However, a global interpretation is recommended, and the professional must decide regarding the clinical context of the patient. For example, furosemide is considered anticholinergic in four of the scales included in $\mathrm{ABC}$, however, in three of them the potential is low or null. Furosemide is a frequently prescribed drug, so it is not appropriate to consider that it is the cause of the anticholinergic risk individually. If the total load is high, it could be useful to optimize treat- ment by intervening in furosemide due to the cumulative effect of anticholinergic drugs. On the other hand, suggestions for changes in treatment are included to reduce anticholinergic burden. From this, an accurate and upto-date report of suggestions on pharmacological treatment to reduce the risk of anticholinergic events can be generated for each patient (Fig. 3).

2. LESS-CHRON. This tool follows the structure of the LESS-CHRON, initially asking users to select the therapeutic groups included in patient treatment (cardiovascular, genitourinary, etc.). This reduces the options and then only shows criteria that applies specifically to the patient if the conditions are met. Some LESS-CHRON criteria require understanding the patient's cognitive function (according to the Pfeiffer questionnaire [26]), functional function (according to the Barthel index [27]) and / or prognosis (according to the Profund Index [28]). The LESS-CHRON tool incorporates these indexes and can calculate them instantly if needed. Finally, a recommendation report is obtained that includes the medications to be deprescribed, as well as a proposal for the review date of patients (which is included in the LESS-CHRON Agenda) and the health variables to be monitored. Example of a recommendation report in the LESS-CHRON criteria is shown in Fig. 4.

3. TRIGGER-CHRON. This tool supports users to carry out a guided review of the patient's medical records, explore different situations or risks that could occur to each patient. These situations, or triggers, are organ-
Fig. 3 Example of a result with the ABC Calculator

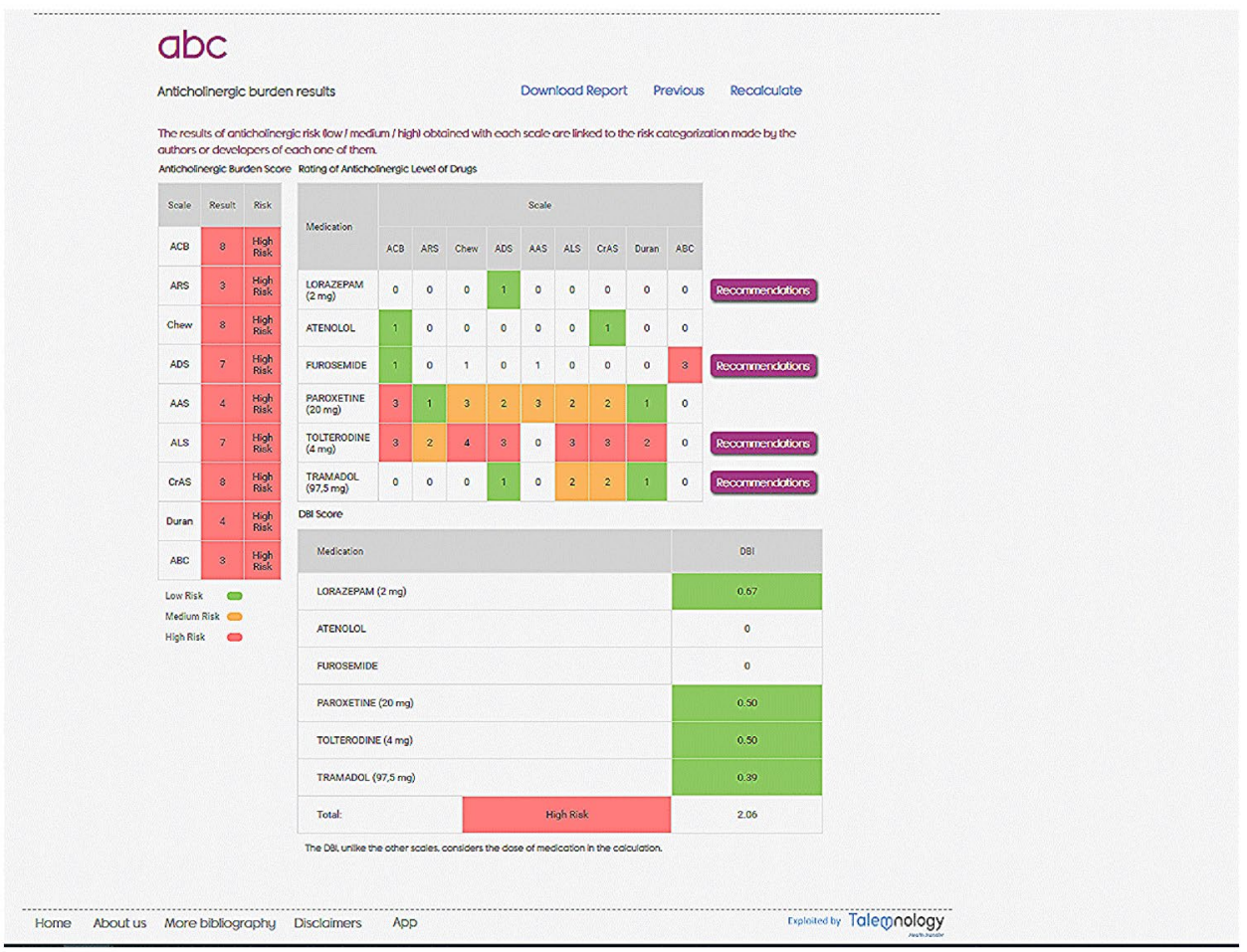


ized in 5 modules: care, antidotes and treatment, plasma concentration, analytical parameters and emergencies., These are shown progressively, and the user should click/mark those that have occurred in the patient. Finally, the tool calculates, based on the positive predictive value, the risk that the patient has of suffering adverse drug events. It also highlights the medication or medications that may be responsible (Fig. 5).

\section{Discussion}

CHRONIC-PHARMA is the result of an innovative research process focused on chronic patients, whose results have been transformed into practical value for clinical practice. The integration of these three tools in a single web/app platform has improved the accessibility of professional support tools. It is one of the most comprehensive web/app platforms currently available that focuses on the optimization and evaluation of pharmacotherapy in a population of chronic patients.

There are tools that have already been published in the literature to support prescriptions aimed at the same type of patients. This is the case of the PRIMA-eDS project and the G-MEDSS tool, between others. These differ from the CHRONICPHARMA tool in their nature. Thus, PRIMA-eDS, apart from being a tool designed to be integrated in a computerized decision support system, is being developed in the context of a randomized clinical trial and consists of a database that allows analysis of patient prescriptions, issuing recommendations [29]. On the other hand, G-MEDSS was developed based on the methodology of a previous tool, DBI calculator, expanding its usefulness by adding other tools through a scenario-based design [5]. CHRONIC-PHARMA has not been validated as a platform, but rather each of the tools it integrates have been developed in different clinical contexts with rigorous methodologies for their creation and with the participation of potential users.

We intend to carry out activities that favor its implementation and visibility, such as its dissemination through clinical sessions, presence in national and international scientific conferences, as well as in scientific societies. Collaboration with clinicians is necessary to promote its use and the involvement of external biotechnology companies (public or private) who can support technical development, scalability, and dissemination of the platform. This is the reason why our research group is currently carrying out multicenter projects in which the tools included in the platform are used to

\section{less-chron}

Previous Download Report Recalculate

After reviewing your pharmacological treatment according to your diagnoses following the LESS-CHRON criteria, it is advisable to consider the description of ANTIHYPERTENSIVES, which is prescribed in its treatment, indicated for: high blood pressure. The proposed deprescribing criteria is met: patient older than 80 years, with systolic blood pressure $\varsigma 160 \mathrm{mmhg}$ and more than one antihypertensive drug, withdraw one of them. To do this, we will monitor that there has not been blood pressure. Your next review will be approximately within a perio 0 of: 3 months.

After reviewing your pharmacological treatment according to your diagnoses following the LESS-CHRON criteria, it is advisable to consider the description of ANTICHOLINERGICS, which is prescribed in its treatment indicated for urinary incontinence. The proposed ceprescribing criteria is met: use of nappy. To do this, we will monitor that there has not been urine control. Your next review will be approximately within a period of: 1 month.

After reviewing your pharmacological treatment according to ycur diagnoses following the LESS-CHRON criteria, it is advisable to consider the description of: ANTICHOLINERGICS, which is prescribed in its treatment, indicated for. urinary incontinence. The proposed deprescribing criteria is met. worsening of dementia symptoms in patients under anticholinesterase treatment. To do this, we will monitor that there has not been urine control. Your next review will be approximately within a period of: 1 month.

After reviewing your pharmacological treatment according to your diagnoses following the LESS-CHRON criteria, it is advisable to consider the description of: BENZODIAZEPINES, which is prescribed in its treatment, indicated for: anxiety. The proposed deprescribing criteria is met: absence of anxiety in the previous month. To do this, we will monitor that there has not been monitoring components of anxiety. Your next review will be approximately within a geriod of: 1 month.

Barthel results

Not calculated

Pfeiffer results

Not calculated

Profund results

Not calculated

Home Aboutus Morebibliography Disclaimers App

Fig. 4 Example of report with the LESS-CHRON tool 
Fig. 5 Example of triggers with the TRIGGER-CHRON tool

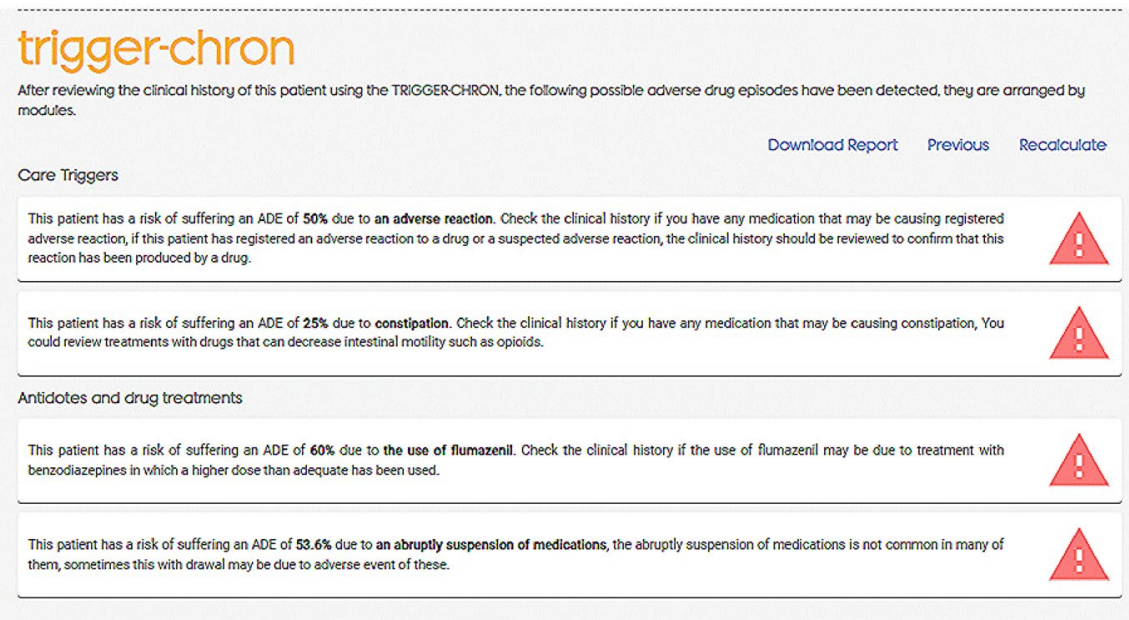

support the review of medications by geriatricians, internists and pharmacists from hospitals, primary care and nursing homes. The main strength of this project is the development of an innovative platform that could be used by healthcare professionals to optimize the pharmacotherapy in this specific, yet ever-expanding group of patients.

CHRONIC-PHARMA is presented as a fast, simple, free access and attractive platform, available in Spanish and English, which translates into greater global visibility. It offers a single record of patient data as well as obtaining a final report of recommendations in each of the tools. It allows users to register the patients and save their information in a safe way (password access and encrypted patient data), useful for clinical practice and research.

We hope that the web/app will make it easier for prescribers to review the medication of chronic patients. Its implementation in clinical practice can help to quickly detect drugs that can be suspended or modified to reduce the risk of ADE, as well as to quickly detect drug-related adverse events. This would decrease the pharmacological burden in these patients, who usually take five drugs or more per day [30], and ultimately improve safety.

We consider that this platform is capable of being incorporated into clinical practice guidelines and protocols aimed at these patients. However, the platform is not currently planned to be integrated into the clinical history computer systems, as it is a complex process that requires more experience and institutional support.

The first version of $\mathrm{ABC}$ has already had great impact, with a total of 5,000 registrations to the platform, half of them registered in 2019. Users are based primarily in Spain, UK, USA, France, Australia and Canada. In 2019, 21,500 calculations were made with 13,110 new patient files and around 181,000 visits to the website. There have also been numerous publications that have referenced the tools that make up this platform [31-37]. In the most recent reviews on this topic, LESS-CHRON is referenced as a powerful tool to be used amongst the chronic patient $[34,35]$. The quick and widespread acceptance of these tools encourages us to promote this platform more widely in the healthcare environment, as well as to continue developing our research work to implement new tools and update current ones.

The platform aims to be a portal that can be extended to incorporate further tools developed with a rigorous methodology focused on improving the safety and pharmacotherapy of the chronic patient, such as tools to improve compliance, review of adequacy, reconciliation, or drug-drug interactions. One limitation to this platform is that it is not capable of extracting the information from patient's clinical history directly, and healthcare professionals must input the data manually.

As for the treatment modification recommendations included in ABC, are based on a systematic review carried out by the working group whose objective was to ascertain the available scientific evidence on actions carried out to reduce a high anticholinergic load in the elderly. The work to keep these recommendations updated is continuous and any changes to recommendations will be incorporated in the next update. Thanks to this new version, support in decision-making is offered to optimize treatment in relation to anticholinergic risk.

The platform can be applied systematically to chronic patients to detect drugs that present increased risk to patients, rather than benefits. However, to apply the LESS-CHRON criteria, we recommend complementing the results with a clinical interview. In some cases, it is also necessary to calculate other scales such as the Barthel Index or the Pfeiffer Scale. To simplify these calculations, access to these scales has been provided directly on the platform. In addition, the platform allows users to set alerts on the recommended date to review the success or failure of the prescription. The TRIGGER-CHRON tool is the only list of triggers for chronic and multimorbid 
elderly patients which allows identifying adverse drug event and their possible prevention. It has shown that from every 4 reviewed medical records an adverse drug event can be detected.

In terms of applicability, it is a platform that is useful at both hospital and primary care levels. In the case of a hospital admission, it should be a priority to use the TRIGGER-CHRON to detect patients at risk of suffering adverse drug events. Subsequently, it is also useful to apply the two remaining tools on anticholinergic burden and deprescription for the comprehensive assessment of pharmacotherapy of chronic patients at time of discharge. On the other hand, the systematic use of the platform in primary care could help in the review of polymedicated patients to reduce adverse events associated with medication. The benefits could be both short and long term. Its use is also extendable to physicians and pharmacists, among other health professionals. It is expected to have an impact not only on health by improving patient safety, but also at the organizational level, providing uniformity in decision making and clinical judgment. Finally, it is possible that the reduction of polytherapy in appropriate cases, will lead to a reduction in costs of the drugs and derived from possible medical visits or hospitalizations.

It is therefore expected that the CHRONIC-PHARMA platform will have a high acceptance by the scientific and healthcare community, as the combined use of the three tools through a user-friendly, digital interface is more comprehensive, efficient and easily accessible.

\section{Conclusions}

In conclusion, the newly developed CHRONIC-PHARMA web and mobile app for chronic patients is a potentially useful platform in the modern technological era. CHRONIC-PHARMA integrates three useful tools into a single portal, for the optimization of pharmacotherapy of chronic patients.

The app is available in the Google Play store and both the website and app are free and can be shared and distributed among the healthcare community.

Acknowledgements This study was supported by the health research project (exp N $\left.{ }^{\circ} \mathrm{PI}-0195-2016\right)$ and the innovation research project LESS-PHARMA-CHRON (PIN-0204-2018) from the Health Council of the Andalusian Regional Government, Spain.

Funding Open Access funding provided thanks to the CRUE-CSIC agreement with Springer Nature. The Health Council of the Andalusian Regional Government (Spain) supported this work within the 2018 by an innovation project ( $\mathrm{N}^{\mathrm{o}}$ exp. PIN- 0204-2018).

Code Availability (Software Application or Custom Code) Protected custom code.

\section{Declarations}

Conflict of Interest The authors declare no conflict of interest.

Open Access This article is licensed under a Creative Commons Attribution 4.0 International License, which permits use, sharing, adaptation, distribution and reproduction in any medium or format, as long as you give appropriate credit to the original author(s) and the source, provide a link to the Creative Commons licence, and indicate if changes were made. The images or other third party material in this article are included in the article's Creative Commons licence, unless indicated otherwise in a credit line to the material. If material is not included in the article's Creative Commons licence and your intended use is not permitted by statutory regulation or exceeds the permitted use, you will need to obtain permission directly from the copyright holder. To view a copy of this licence, visit http://creativecommons.org/licenses/by/4.0/.

\section{References}

1. Süle A, Miljković N, Polidori P, Kohl S (2019) Position paper on an ageing society. Eur J Hosp Pharm 26(6): 354-356. https:// doi.org/10.1136/ejhpharm-2019-001910.

2. O'mahony D, O'sullivan D, Byrne S, O'connor MN, Ryan C, Gallagher P (2015) STOPP/START criteria for potentially inappropriate prescribing in older people: version 2. Age Ageing 44 (2): 213-8. https://doi.org/10.1093/ageing/afu145.

3. Samuel MJ (2015) American Geriatrics Society updated Beers criteria for potentially inappropriate medication use in older adults. J Am Geriatr Soc 63 (11): 2227-46. https://doi.org/10. 1111/jgs. 13702.

4. O'Mahony (2020) D. STOPP/START criteria for potentially inappropriate medications/potential prescribing omissions in older people: origin and progress. Expert Rev Clin Pharmacol. 2020 Jan;13(1):15-22. https://doi.org/10.1080/17512433.2020. 1697676.

5. Kouladjian O'Donnell L, Reeve E, Hilmer SN.(2021) Development, validation and evaluation of the Goal-directed Medication review Electronic Decision Support System (G-MEDSS)@ Res Social Adm Pharm. 2021 Sep 14:S1551-7411(21)00338-7. https://doi.org/10.1016/j.sapharm.2021.09.004.

6. Scott IA, Pillans PI, Barras M, Morris C. (2018) Using EMRenabled computerized decision support systems to reduce prescribing of potentially inappropriate medications: a narrative review. Ther Adv Drug Saf. 2018 Jul 12;9(9):559-573. https:// doi.org/10.1177/2042098618784809.

7. Rudolph JL, Salow MJ, Angelini MC, McGlinchey RE (2008) The anticholinergic risk scale and anticholinergic adverse effects in older persons. Arch Intern Med 168 (5): 508-13. https://doi.org/10.1001/archinternmed.2007.106.

8. Boustani M, Campbell N, Munger S, Maidment I, Fox C (2008) Impact of anticholinergics on the aging brain: a review and practical application. Aging Health 4: 311-20, 2008. https://doi. org/10.2217/1745509X.4.3.311.

9. Villalba-Moreno AM, Alfaro-Lara ER, Pérez-Guerrero MC, Nieto-Martín MD, Santos-Ramos B (2016) Systematic review on the use of anticholinergic scales in poly pathological patients. Arch Gerontol Geriat. 64:178-80, 2016. https://doi. org/10.1016/j.archger.2015.10.002.

10. Villalba-Moreno Á, Alfaro-Lara ER, Sánchez-Fidalgo S, SantosRamos B (2017) Development of the Anticholinergic Burden Calculator Web Tool. Farm Hosp 41(5):647-648. https://doi.org/10.7399/ fh.10799. 
11. Rodríguez-Pérez A, Alfaro-Lara ER, Nieto-Martín MD, RuizCantero A, Santos-Ramos B (2015) Deprescribing in patients with multimorbidity: A necessary process. Eur J Intern Med 26(7):e18-19. https://doi.org/10.1016/j.ejim.2015.06.011.

12. Rodríguez-Pérez A, Santos-Ramos B, Alfaro-Lara ER (2017) Deprescribing: guiding its definition. Farm Hosp 41(6):698-699 https://doi.org/10.7399/fh.10848.

13. Farrell B, Pottie K, Thompson W, Boghossian T, Pizzola L,. Rashid FJ, et al (2017) Deprescribing proton pump inhibitors: Evidence-based clinical practice guideline. Can Fam Physician 63(5):354-64. PMID:28500192.

14. Pottie K, Thompson W, Davies S, Grenier J, Sadowski CA, Welch V, et al (2018) Deprescribing benzodiazepine receptor agonists: Evidence-based clinical practice guideline. Can Fam Physician 64(5):339-51. PMID: 29760253.

15. Lavan AH, Gallagher P, Parsons C, O'Mahony D (2017) STOPPFrail (Screening Tool of Older Persons Prescriptions in Frail adults with limited life expectancy): consensus validation. Age Ageing 46(4):600-7. https://doi.org/10.1093/ageing/afx005.

16. Nyborg G, Straand J, Klovning A, Brekke M (2015) The Norwegian General Practice - Nursing Home criteria (NORGEP$\mathrm{NH}$ ) for potentially inappropriate medication use: A web-based Delphi study. Scand J Prim Health Car. 33:134-141. https://doi. org/10.3109/02813432.2015.1041833.

17. Rodríguez-Pérez A, Alfaro-Lara ER, Albiñana-Perez S, NietoMartín MD, Diez-Manglano J, Pérez-Guerrero MC (2017) Novel tool for deprescribing in chronic patients with multimorbidity: List of Evidence-Based Deprescribing for Chronic Patients criteria. Geriatr Gerontol Int 17:2200-7 https://doi.org/10.1111/ggi.13062.

18. Rodríguez-Pérez A, Alfaro-Lara ER, Sierra-Torres MI, VillalbaMoreno Á, Nieto-Martín MD, Galván-Banqueri M (2019) Validation of the LESS-CHRON criteria: reliability study of a tool for deprescribing in patients with multimorbidity. Eur J Hosp Pharm 26(6):334-338. https://doi.org/10.1136/ejhpharm-2017-001476.

19. Resar RK, Rozich JD, Classen D (2003) Methodology and rationale for the measurement of harm with trigger tools. Qual Saf Health Car. 12(suppl 2):ii39-ii45. https://doi.org/10.1136/ qhc.12.suppl_2.ii39.

20. Griffin FA, Resar RK IHI Global Trigger Tool for Measuring Adverse Events. In: 2nd ed. Cambridge MA: Institute for Healthcare Improvement: IHI Innovation Series White Paper; 2009: Available at: http://www.ihi.org/resources/Pages/Tools/ IHIGlobalTriggerToolforMeasuring AEs.aspx. [accessed 2020-03-09].

21. Health Quality \& Safety Commission (2016) The global trigger tool: A review of the evidence. Wellington: Health Quality \& Safety Comission.

22. Toscano-Guzmán MD, Galván-Banqueri M, Otero MJ, AlfaroLara ER, Casajus-Lagranja P, Santos-Ramos B (2017) Development of a trigger tool to identify adverse drug events in elderly patients with multimorbidity. J Patient Saf https://doi.org/10. 1097/PTS.0000000000000389.

23. Toscano-Guzmán MD, Galván-Banqueri M, Otero MJ, SánchezFidalgo S, Font-Noguera I, Pérez-Guerrero MC.(2018) Validating a Trigger Tool for Detecting Adverse Drug Events in Elderly Patients With Multimorbidity (TRIGGER-CHRON). J. Patient. Saf. 2018. https://doi.org/10.1097/PTS.0000000000000552.

24. Statista. New York NY: Statista; 2015 Jan. Year-on-year growth in time spent per mobile app category in 2014 http:// www.statista.com/statistics/251096/fastest-growing-shoppingapp-categories/ [accessed 2020-03-09] [WebCite Cache ID $6 \mathrm{Vct} 3 \mathrm{XDGV}]$
25. Kouladjian L, Gnjidic D, Chen TF, Hilmer SN.(2016) Development, validation and evaluation of an electronic pharmacological tool: The Drug Burden Index Calculator@. Res Social Adm Pharm. 2016 Nov-Dec;12(6):865-875. https://doi.org/10.1016/j. sapharm.2015.11.002. Epub 2015 Nov 11.

26. Pfeiffer E.(1975) A short portable mental status questionnaire for the assessment of organic brain deficit in elderly patients. J Am Geriatric Soc 1975; 23: 433-441. https://doi.org/10.1111/j. 1532-5415.1975.tb00927.x.

27. Mahoney FI, Barthel DW. Functional evaluation: the Barthel Index. Md State Med J 1965;4:61-5. PMID: 14258950

28. Bernabeu-Wittel M, Ollero-Baturone M, Moreno-Gaviño L et al. (2011) Development of a new predictive model for paleopathological patients. The PROFUND index. Eur J Intern Med. 2011;22:311-17. https://doi.org/10.1016/j.ejim.2010.11.012.

29. Sönnichsen A, Trampisch US, Rieckert A, Piccoliori G, Vögele A, Flamm M, Johansson T, Esmail A, Reeves D, Löffler C, Höck J, Klaassen-Mielke R, Trampisch HJ, Kunnamo I.(2016) Polypharmacy in chronic diseases-Reduction of Inappropriate Medication and Adverse drug events in older populations by electronic Decision Support (PRIMA-eDS): study protocol for a randomized controlled trial. Trials. 2016 Jan 29;17:57. https:// doi.org/10.1186/s13063-016-1177-8.

30. Rollason V, Vogt N (2003) Reduction of polypharmacy in the elderly: a systematic review of the role of the pharmacist. Drugs Aging 20:817-32 2003. https://doi.org/10.2165/ 00002512-200320110-00003.

31. Hernández M, Mestres C, Modamio P, Junyent J, Costa-Tutusaus, Lastra CF, et al. (2019) Adverse Drug Events in Patients with Dementia and Neuropsychiatric/Behavioral, and Psychological Symptoms, a One-Year Prospective Study. Int J Environ Res Public Health 15;16(6):934. https://doi.org/10.3390/ijerph16060934.

32. Joung KI, Shin JY, Cho SI (2019) Features of anticholinergic prescriptions and predictors of high use in the elderly: Populationbased study. Pharmacoepidemiol Drug Saf 28(12):1591-1600. https://doi.org/10.1002/pds.4902.

33. Tiisanoja A, Syrjälä AMH, Kullaa A, Ylöstalo P (2020) Anticholinergic Burden and Dry Mouth in Middle-Aged People. JDR Clin Trans Res 5(1):62-70. https://doi.org/10.1177/ 2380084419844511.

34. Reeve E (2020) Deprescribing tools: a review of the types of tools available to aid deprescribing in clinical practice. J Pharm Pract Res 50:98-107. https://doi.org/10.1002/jppr.1626.

35. Thompson W, Lundby C, Graabaek T, Nielsen DS, Ryg J, Søndergaard J, et al (2019) Tools for Deprescribing in Frail Older Persons and Those with Limited Life Expectancy: A Systematic Review. J Am Geriatr Soc 67:172-180. https://doi.org/10.1111/jgs.15616.

36. Hu Q, Qin Z, Zhan M, Chen Z, Wu B, Xu T (2020) Validating the Chinese geriatric trigger tool and analyzing adverse drug event associated risk factors in elderly Chinese patients: A retrospective review. PLoS One 15(4): e0232095. https://doi.org/ 10.1371/journal.pone.0232095.

37. Weingart SN, Nelson J, Koethe B, Yaghi O, Dunning S, Feldman A, et al (2020) Developing a cancer-specific trigger tool to identify treatment-related adverse events using administrative data. Cancer Med 9(4):1462-1472. https://doi.org/10.1002/cam4.2812.

Publisher's Note Springer Nature remains neutral with regard to jurisdictional claims in published maps and institutional affiliations. 